\title{
Anorexia Nervosa: The physiological consequences of starvation and the need for primary prevention efforts.
}

\author{
Michael Sidiropoulos*
}

\section{CASE PRESENTATION}

A 17-year-old girl was brought to the emergency department by her worried parents. The patient was described by her parents as having symptoms of fatigue, dizziness and syncope over the past few days. She also had no appetite, a weight loss of $11.4 \mathrm{~kg}$ over the past four months and a recent uncharacteristic pursuit to exercise. When the patient was further questioned, she complained of cold hands and feet, constipation, dry skin and hair, and headaches. Her social history revealed social withdrawal, depression, and irritability, as well as having difficulty concentrating and making decisions. Her performance at school had also fallen. The physical exam revealed a quiet, young girl who looked her age, was in no distress, but had cool peripheries. Her skin was slightly mottled in appearance, with some anhydrotic eczema of her extremities, and her blood pressure was difficult to auscultate. When obtained, it was lower than her usual values, at 84 systolic and 70 diastolic. Her pulse was 47 $\mathrm{bpm}$, and irregularly irregular. Respiratory and abdominal examinations were normal. The patient however looked very underweight and thin. Her weight and height were $47 \mathrm{~kg}$ and $1.7 \mathrm{~m}$, respectively; her calculated body-mass index (BMI) was 16 . She also had a moderate loss of muscle mass and edema of her extremities. Further history taking from the patient, while alone, revealed that she had stopped menstruating seven months ago. There were no changes in sleep or any changes in bladder or bowel movements, and nausea and vomiting were denied.

Her past medical history was remarkable for a stress fracture of her fibula and multiple admissions to hospital for depression, and she was also once admitted for suicidal ideation. Moreover, the patient denied any

*To whom correspondence should be addressed:

Michael Sidiropoulos

3350 Kingston road, Toronto, Ontario, M1M 1R2.

E-mail: michael.sidiropoulos@utoronto.ca current laxative or diuretic use, and when asked about her diet, she stated that it consisted of yogurt and some fruit, with no meal at lunchtime while at school. Daily servings would not exceed a total of $750 \mathrm{kcal} /$ day and her meals never contained any fat. She described herself as "fat...overweight" and her only pastime was exercising. When asked about her weight, she stated, "the more weight I lose, the more I wish to loose." She denied having any problems and explained that she was annoyed that her parents, friends, and teachers were concerned. It seems that the patient has very poor insight into her situation, and continues to perceive herself as healthy. Despite being aware that she is underweight, amenorrheic and bradycardic, she feels that she does not need to be in hospital.

The social history revealed that the patient lives with her parents and two older siblings. When asked how her relationship is with her siblings, the patient replied, "I have never felt close to anyone..." The patient is a grade 12 student, and has considerable pressure from her parents to be an A student, and that they have very high expectations for her. She was involved in numerous extracurricular activities at school in the past; however, she is now preoccupied with her exercise goals, and has little time for other activities. English is a second language for the parents of the patient and there have been numerous problems in communication. There were parent-child relationship problems evident immediately on presentation. The mother has been unable to get the patient to eat at home; however, the family has been reluctant to have the patient admitted to hospital numerous times in the past. Family history revealed that a first cousin from the mother's side had been diagnosed with anorexia nervosa while in her teens.

Laboratory values revealed anemia, leucopenia, hypoglycemia and numerous electrolyte disturbances. Her specific gravity in her urine was 1.005 , indicating that she may have attempted to water load prior to 
being brought to the emergency department by her parents, and that her weight may in fact be lower than measured.

\section{THE PHYSIOLOGICALCONSEQUENCES OF THE STARVATION}

The prevalence rates of anorexia nervosa (AN) have been shown to vary between 0 and $0.9 \%$, with an average rate of $0.29 \%$ in young females, according to the Diagnostic and Statistical Manual of Mental Disorders, Fourth Edition (DSM-IV) criteria (1-3). The DMS-IV criteria for AN is as follows (3):

A. Refusal to maintain body weight at or above a minimally normal weight for age and height

B. Intense fear of gaining weight or becoming fat, even though underweight

C. Disturbance in the way in which one's body weight or shape is experienced, undue influence of body weight or shape on self-evaluation, or denial of the seriousness of the current low body weight

D. In postmenarcheal females, amenorrhea

In addition, there are different types of AN. The restricting type of $\mathrm{AN}$, and the binge-eating/purging type. The former defines a person who has not regularly engaged in binge-eating or purging behavior, such as self-induced vomiting or the misuse of laxatives, while the latter does (3).

The pathogenesis of AN cannot be easily explained, but a combination of biologic, psychological and social factors most likely contribute. Lifestyle issues, such as nutrition and physical activity, are of paramount importance and form the basis for both pathogenesis and treatment issues. The identified and potential role of both nutrition and physical activity in the pathogenesis of this case can easily be seen with most patients who suffer from anorexia nervosa. The patient in this case clearly suffers from anorexia nervosa, and is characterized by determined dieting, phobic avoidance of certain foods that contain fat, a pervasive fear of calories, fat and fatness, as well as having a disturbed body image and a heightened desire to lose more weight. The patient suffers from the restricting type of AN, which is characterized by marked caloric reduction, typically to $400-700 \mathrm{kcal}$ per day, which is often accompanied by compulsive exercise. The patient subsequently suffers from her lack of nutrition and over-exercising, as she begins to have numerous symptoms, such as fatigue, weakness, syncope, and amenorrhea, which are directly related to her lack of eating and strenuous exercising habits.

Adolescence is a time of major growth and development, and the role that nutrition plays cannot be more emphasized. The pubertal growth spurt accounts for approximately $25 \%$ of adult height, $50 \%$ of adult weight, and a foundation for the development of subsequent reproductive capacity (4). Nutritional intake at this time has to be suitable, in order to support this growth. Nutrition not only provides the energy for this growth, but the proper balance of proteins, carbohydrates, fats, vitamins, and minerals. The Recommended Daily Allowance (RDA) standards for adequate nutrition have been developed for adults, but not for adolescents. In addition, most of these recommendations have to be adjusted for special conditions such as a significant growth spurt, athletic participation, major stress or a chronic illness (5). According to one source, caloric requirements for adolescents aged $15-18$ are $40 \mathrm{kcal} / \mathrm{kg}$, with $55-60 \%$ derived from carbohydrates, and less than $30 \%$ from fats (6). Inadequate nutrition and excessive exercising during these "opportunity" years may lead to numerous complications, both acute and chronic, as seen in our patient (7).

Medical complications resulting from semistarvation and overexercising affect virtually every organ system. Common signs and symptoms include loss of subcutaneous fat tissue, orthostatic hypotension, bradycardia, impaired menstrual function, hair loss, and hypothermia. Such symptoms are present within our case. Numerous laboratory measures are affected, among them serum electrolyte levels and thyroid function. Our patient had numerous electrolyte abnormalities, including low calcium and potassium levels. Patient's who have AN in adolescence, as is the case with our patient, have numerous medical complications that may persist into the adult years (7). These include concerns on the bone density of these individuals, and the possibilities of osteopenia and osteoporosis early on in life. Patients may have shorter stature than expected and high rates of stress fractures early on in their lives, such as in their mid-20s (8). Many other complications of AN, such as amenorrhea, are believed to be the result of the severe caloric restriction, which suppresses the hypothalamic-pituitary axis (9). Biochemical mediators of this process include cortisol, leptin, growth hormone, and insulin-like growth factor I, all essential mediators of growth and development in an adolescent (10). This suppression results in a block in the production of luteinizing hormone and follicle-stimulating hormone. Without these hormones and their normal cycling patterns, there is no production of estrogen and no ovulation, and subsequent amenorrhea. The amenorrhea may sometimes be the reason the patient seeks medical attention. Less commonly, it may be desired by the patient, as in our case, where she seeks to remain 'young (4).' It is this absence of estrogen and amenorrhea which represents a significant "missed opportunity" for bone formation. In an athletic female, as is our case, who strenuously exercises, stress fractures are common. 
Furthermore, the combination of an eating disorder, menstrual changes and osteoporosis has been termed the "female athletic triad (11)."

Adolescence is a critical time not only for bone formation, but the formation of all the organ systems within the body. A critical foundation of cell mass and cell numbers is laid down during these critical years, as the body is a mosaic of hormones and growth and developmental physiological axis's are at their peaks. Skeletal, smooth and cardiac muscle mass is responsive to hormonal changes, and begin to fully develop and expand to its mature form. Underdevelopment and wasting of these systems leads to weakness and all of the cardiac manifestations of $\mathrm{AN}$, as seen in our patient, such as fatigue, generalized weakness, syncope, bradycardia and arrhythmias. Of most concern, is the growth and development of cardiac muscle, as cardiac failure and arrhythmias can result in severe starvation states and can lead to death. This is believed to be due to the loss of critical cardiac muscle mass and subsequent abnormalities in cardiac conduction through electrolyte imbalances from poor nutrition (4).

Changes in cognitive development are also known to occur. Our patient has had some difficulties in school and also in communicating with her parents and friends. In the short-term, the consequences of a diet deficient in calories and nutrients, and excessive strenuous and relentless exercising, can lead to excessive sleeping and deprivation of cognitive and social stimuli, as school, social events and friends are neglected. Furthermore, it is well known in the literature, that the brain loses both white and gray matter during severe weight loss during semistarvation. Furthermore, weight restoration results in the return of white matter to premorbid levels, but some gray matter loss persists (12). These deficits may not be clinically evident initially, but may be associated with long-term effects on cognitive functioning. Another long-term effect, is that women who have had AN also have higher rates of miscarriages and lower infant birth weights than do healthy women (13).

Standardized mortality ratios are elevated in AN patients for all causes of death, especially suicide (14). Suicidal ideation is also common. Death from medical causes is due to primarily starvation or purge-related arrhythmias, as mentioned above. The probability of recovery is known to vary inversely with the severity of weight loss and the presence of coexisting psychiatric disorders, such as depression and personality disorders, which are also very common in AN (15). Moreover, psychosocial factors, such as the transition of adolescence into adulthood, and the acceptance of the physical changes of puberty, peer group involvement and adult autonomy are especially difficult in a teenage female who suffers from weakness, fatigue and irritability, and the symptoms of AN. Therefore, these issues perpetuate the viscous cycle of $\mathrm{AN}$, and contribute to starvation and excessive exercising as the patient is out to "prove" their identity, autonomy, and peer acceptance, without fear and appreciation of the consequences.

\section{MANAGEMENT OF THE PATIENT WITH ANOREXIA NERVOSA}

The physician must obtain a thorough and detailed history and physical. Particular emphasis should be placed on the diet and social history, as well as the past history, in order to delineate the needs of the patient and explore the impact that nutrition and physical activity could have had on the patient's symptoms and current status, as well as her future growth and development. A detailed diet and social history is instrumental in revealing the true pathogenesis. Furthermore, the severity of the patient's condition and issues at hand should also be well described, and the need for an immediate and diverse treatment and management plan put forth. The patient in our case, should be hospitalized because the complications associated with the patient's malnutrition and weight loss are likely to be life-threatening. A physician can adequately explore all the issues within the clinical setting, through progressive interviews and discussions with both the patient and family, both individually and together. An advocacy role should be established, and care should be taken to spend time alone with the patient. A trusting relationship should be formed with both the patient and family, and blame and guilt must be avoided.

Physicians must understand that addressing the full concerns of the patient with anorexia nervosa would need a multifaceted and multidisciplinary approach. Referrals from both a psychiatrist and a dietician should be made. A clinical team can be very effective in addressing the nutrition and physical activity issues, as well as other biologic, psychological, and social issues related to the patient's condition. Weight should be monitored closely throughout the patient's admission, as well as vital signs and urine specific gravity. Regular physical examinations should also be done, as well as recording food intake and calories.

The biologic aspects of treatment are aimed at early improvement of the physiologic state of the patient. These include a healthy goal for weight and range that should be determined by a dietician. The main goal is to stop further weight loss and prevent relapses through establishing a highly structured pattern of three meals and one to three snacks a day. When the caloric needs are determined, an individualized meal plan should be developed. The dietician, along with the clinical team can effectively: 1) educate the patient and family regarding nutrient needs; 2) develop a balanced meal 
plan with a target caloric range to achieve the weight and range goals put forth; 3 ) assess diet journals; and 4) provide feedback (16).

Education plays an important part in the treatment of patients with anorexia nervosa, as many adolescents with AN have misconceptions about what constitutes "healthy eating (16)." Cognitive-behavioral therapy (CBT) can be instituted in order to try and break, as well as restructure the strict food rules and rituals, and erroneous beliefs that the patient may have. Numerous studies have shown that CBT was significantly more effective than nutritional counseling in improving outcome and preventing relapse in AN (17-19). In addition, the patient can also be formally assessed for mood disorders, such as depression and anxiety. Furthermore, patient's conditions can be placed in a developmental framework in order to tailor the patient's treatment program to their psychosocial development. Referral's to eating disorder clinics, such as at the Hospital for Sick Children's, can also be made upon discharge of the patient for follow-up.

Finally, communication with the family doctor of the patient's is essential. By contacting the family doctor during a patient's admission, the family doctor can be made aware of the situation and the patient's status. A follow-up appointment should be made, thereby helping the family physician organize and set-up further specialist follow-up appointments and ensure continuity of care.

\section{PRIMARY PREVENTION OF ANOREXIA NERVOSA}

There are numerous actions that the physician, along with the family, allied health care workers and/or through a broader public health initiative can accomplish in this particular case that will have longstanding implications on the patient's future development and growth and will increase the likelihood of healthy outcomes through primary prevention. Physicians involved in primary prevention efforts have the potential to reach large and diverse groups of adolescents who may be at risk of disordered eating or progression to better characterized eating disorders. Moreover, halting the severity of an eating disorder, or preventing the progression of an eating disorder to include other psychiatric comorbidities, such as mood disorders, could be accomplished. The need for primary prevention efforts, particularly by a physician, could not be more evident. Given the high prevalence of poor eating habits and eating disorders, the broad range of young people affected, their short and long-term health consequences, their resistance to treatment, and their cost, there is a clear justification for primary prevention (20). Different factors and strategies will need to be addressed and implemented in different settings, such as in the community, schools, or clinical setting.

Primary prevention can take place at numerous levels, from social interventions to individuals focuses. Each visit to a physician's office should be effectively utilized as a window of opportunity for identifying problems early and also counseling patients and parents about eating disorders in the appropriate age groups. Allowing the patient more opportunities for in-depth and intimate discussions on issues such as body image and self-esteem, with a more comprehensive focus would be ideal. Furthermore, awareness of the disorders and how to prevent them, how to notice them early, and how to seek help, should be done. Furthermore, using part of a check-up visit to inquire about school, family, nutrition, and extracurricular activities can identify that problems are developing or provide an outlet for adolescents to discuss problems. Discussions with the adolescent alone and the parents alone could provide insight into the home and school environment. In addition, interactions with school teachers could also be done (20).

Effective communication is also essential in primary prevention. Effective communication between tertiary care hospitals, primary care hospitals and community pediatricians and family physicians is essential. This is especially true for patients that are immigrants and unaware of how the health system functions. Effective communication between the health care hierarchy can allow primary care physicians to follow their patients and help coordinate and organize follow-up, thereby decreasing the probability of relapses and adverse outcomes.

Moreover, friends and family members observe situations that are deteriorating, but are unable to proceed as they do not know what to do. Public information help lines could be established where individuals could call anonymously to seek support and advice on how to deal with situations (20). These lines could be established through the health care system, social systems or linked to already existing 'help lines.'

Through a broader public health initiative, a physician can simply participate in a 1-hour presentation on eating disorders to students. Such a presentation can realistically be expected to increase the awareness of the early signs of AN, and can provide phone numbers for referral. In addition, implementation of broad-based primary prevention programs in schools would be effective. Schools are an ideal site for primary prevention programs, as they house a large, captive, potentially at-risk audience, and they provide an environment that harbors learning and positive peer interactions (21). Issues that a physician in a public 
health initiative could address in a school setting could include: reducing body dissatisfaction, critical thinking about sociocultural and peer norms, understanding physical development, improving knowledge about nutrition, diet, and weight control, and skill development, specifically in food selection and preparation, physical activity and dealing with harmful peer pressure through role playing and group discussions. Furthermore, the school environment can act as the site for the initial identification of high-risk adolescents or families, and referral of adolescents and their families for treatment and ongoing support. Some schools can also offer preliminary counseling to youths and their families in association with physicians (20) Moreover, these issues can also be further discussed in a clinical setting.

These primary prevention efforts should not just be aimed at adolescents, but also younger children, as current literature describes that the roots for disturbed eating are known to begin prior to adolescence. Researchers argue that cultural norms for attractiveness are acquired by children at a very young age and that girls associate thinness with attractiveness well before puberty (22). Therefore, all age groups would be targeted for primary prevention, with appropriate content being offered at each stage of the life cycle (23). However, the main focus of primary prevention efforts should be initially directed towards 11-14 year olds, as this age group is in the adolescent transition period which includes puberty, great peer influences, new social roles and significant cognitive development and growth.

Furthermore, even more focused primary prevention efforts may target groups at high risk for AN, and eating disorders, such as gymnasts, dancers, and other athletes. Recently, numerous small-group primary prevention activities, which involved physicians, have been described. These include ballet school programs and programs within college women's facilities. Our patient was highly active in athletics prior to becoming ill (20).

Physicians should advocate for easily accessible educational programs and support for patients with eating disorders. Greater access to eating disorder treatment programs will facilitate the identification and treatment of AN individuals. In this case, a newly immigrated family to Canada, which spoke little English, except for their children, was unknowledgeable about the health care system and what it had to offer. The provision of information regarding available community supports, such as afterschool groups, and health care programs in primary care offices and community clinics which offer eating disorder support, advice, and direction to access other regional and health care services and supports is crucial in a physician. Information can be provided in different forms, such as multilingual pamphlets and posters, or through direct one-to-one discussions at routine visits with patients or parents. A knowledgeable physician that can direct his or her patients to these community and health care services can be an asset to patients.

National interventions could possibly include legislation against false and harmful weight control products, controls on food that is served at schools and improvements in health care access to nutritional and psychological counseling. Social changes include attempts to modify the cultural norms regarding eating behaviors and desired body size. These include difficult but potentially high yield actions of reforming the diet industry and challenging advertising and media to change, particularly that directed to young women. Such changes have started to take place. Hues magazine is "a national magazine for women of all cultures, shapes and lifestyles," and the magazine Seventeen, which is aimed at youths, has been increasing their responsibility regarding stereotyped body images. Numerous physicians take part in their editorials, and have contributed to changing the image and view of the ideal female (18). Community-level programs, which can be integrated with the school system, clinics, local media and religious, youth, and parenting organizations, can be the most influential mode of support and change, and can provide the opportunity to create a synergistic effect among the many different levels and agents of change (20)

Finally, greater access to eating disorder education and treatment programs for adolescents and families will facilitate in the identification and treatment of disordered eating early, and also prevent relapses and life-threatening consequences in patients who already have AN.

\section{REFERENCES}

1. Hoek HW et al. Review of the Prevalence and Incidence of Eating Disorders. Int J Eat Disord 2003; 34:383-396.

2. Hoek HW et al. Incidence, Prevalence and Mortality of Anorexia Nervosa and other Eating Disorders. Curr Opin Psych 2006; 19:389-394.

3. American Psychiatry Association. Diagnostic and Statistical Manual of Mental Disorders-Fourth Edition, Text Revision. Washington, D.C. American Psychiatric Publishing Inc., 2000.

4. Shafer MB, Irwin CE. The adolescent patient. In: Rudolph AM, editor. Rudolph's pediatrics. 19th. Englewood Cliffs (NJ): Appleton \& Lange; 1991 p. 39.

5. Seidenfeld $\mathrm{ME}$ et al. Nutrition and Eating Disorders in Adolescents. Mt. Sinai J Med 2004; 3:155-161.

6. Subcommittee on the Tenth Edition of the RDAs. Recommended Dietary Allowances. Washington (DC): National Academy Press; 1989.

7. Johnson JG et al. Eating Disorders During Adolescence and the Risk for Physical and Mental Disorders During Early Adulthood. Arch Gen Psychiatry 2002; 59:545-552. 
8. Vestergaard $\mathrm{P}$ et al. Fractures in Patients with Anorexia Nervosa, Bulimia Nervosa, and Other Eating Disorders - A nationwide register study. Int J Eat Disord 2002; 32:301-308.

9. Golden NH et al. Resumption of Menses in Anorexia Nervosa. Arch Pediatr Adolesc med 1997; 151:16-21.

10. Audi $\mathrm{L}$ et al. Leptin in Relation to Resumption of Menses in Women with Anorexia Nervosa. Mol Psychiatry 1998; 4:544547.

11. Otis CL et al. American College of Sports Medicine Position Stand. The Female Athlete Triad. Med Sci Sports Exerc 1997; 29:i-ix.

12. Lambe Ek et al. Cerebral Gray Matter Volume Deficits after Weight Recovery from Anorexia Nervosa. Arch Gen Psychiatry 1997; 54:537-542.

13. Bulik CM et al. Fertility and Reproduction in Women with Anorexia Nervosa: A controlled study. J Clin Psychiatry 1999; 60:130-135.

14. Harris EC et al. Excess Mortality of Mental Disorder. Br J Psychiatry 1998; 173:11-53.

15. Westen D et al. Personality Profiles in Eating Disorders: Rethinking the distinction between axis I and II. Am J Psychiatry 2001; 158:547-562.
16. Kreipe $\mathrm{R}$ et al. Eating Disorders in Adolescents and Older Children. Pediatrics in Review 1999; 20:12.

17. Pike KM et al. Cognitive Behavior Therapy in the Posthospitalization Treatment of Anorexia Nervosa. Am J Pyschiatry 2004; 11:2046-2049.

18. Cooper MJ. Cognitive Theory in Anorexia Nervosa and Bulimia Nervosa: Progress, development and future directions. Clin Psychol Rev 2005; 4:511-531.

19. Ball et al. A Randomized Controlled Study of Cognitive Behavior Therapy and Behavioral Family Therapy for Anorexia Nervosa Patients. Eat Disord 2004; 4:303-314 .

20. Rosen DS et al. Review of Options for Primary Prevention of Eating Disturbances Among Adolescents. J Adolescent Health 1998; 23:354-363.

21. Neumark-Sztainer D. School-based Programs for the Prevention of Eating Disturbances. J Sch Health 1996; 66:6471.

22. Feldman W et al. Culture vs. Biology: Children's attitudes towards thinness and fatness. Pediatrics 1988; 81:190-194.

23. Chitty KK et al. The Primary Prevention Role of the Nurse in Eating disorders. Nurs Clin North Am 1991; 26:789-800 .

24. Miller C. What's too Thin? Seventeen 1996; September:16.

Michael Sidiropoulos, Hon.B.Sc, M.Sc., (MD, 2007), is currently a fourth year medical student at the University of Toronto. Michael obtained his Hon.B.Sc in Immunology, and M.Sc in cancer epigenetics (Pathobiology and Laboratory Medicine), both from the University of Toronto. He is also interested in completing a Ph.D in the near future. His main interests are in dermatology and pathology, with a future career in academic medicine. 